PAEDIATRIC LUNG DISEASE

\title{
The need to redefine non-cystic fibrosis bronchiectasis in childhood
}

\author{
K M Eastham, A J Fall, L Mitchell, D A Spencer
}

Thorax 2004;59:324-327. doi: 10.1136/thx.2003.011577

See end of article for authors' affiliations

......................

Correspondence to: Dr D A Spencer, Department of Respiratory Paediatrics, Freeman Hospital, Newcastle upon Tyne NE' 7DN, UK; david. spencer@ nuth.northy.nhs.uk

Received 10 July 2003 Accepted 26 November 2003
Background: Non-cystic fibrosis (CF) bronchiectasis has previously been reported to be rare and progressive in children living in western societies.

Method: A clinical and radiological review was undertaken of 93 children with non-CF bronchiectasis defined by high resolution computed tomographic (HRCT) scanning presenting to a tertiary paediatric respiratory centre since 1996 .

Results: Cases constituted $9.6 \%$ of all new referrals. Male to female ratio was 2:1. Median age at symptom onset was 1.1 years (range 0-16) and of HRCT diagnosis was 7.2 years (1.6-18.8). The most common referral diagnosis of asthma was refuted in 39 of 45 cases. Associations were previous pneumonic illness $(30 \%)$, immunocompromise (21\%), obliterative bronchiolitis $(9 \%)$, congenital lung abnormality (5\%), chronic aspiration (3\%), eosinophilic oesophagitis (2\%), familial syndrome (2\%), primary ciliary dyskinesia (1\%), and right middle lobe syndrome (1\%). $8 \%$ had two associated diagnoses and $18 \%$ were idiopathic. There was agreement between the chest radiograph and HRCT scan for diagnosis and lobe affected in only five cases (5\%). A repeat HRCT scan in 18 cases at a minimum interval of 18 months showed total resolution of the changes in six, improvement in one, progression in five, and was unchanged in six.

Conclusions: Radiologically defined non-CF bronchiectasis in children is not uncommon. Diagnostic delay is a problem. The most common association is a previous pneumonia. Chest radiography is of little diagnostic value, but resolution is possible on HRCT scanning. Bronchiectasis is currently defined as a condition which is both permanent and progressive. This term is not necessarily appropriate for all paediatric patients for whom we suggest an alternative nomenclature.
B ronchiectasis is a morphological term used to describe abnormal irreversibly dilated and often thick walled bronchi. ${ }^{1}$ This traditional definition evolved from Laënnec's original description in 1819 of ectatic bronchi in pathological specimens and implies that the disease process is lifelong and irreversible. ${ }^{2}$ Since this time the term has been used more widely to describe both clinical and radiological disease entities. However, the precise relationship between the clinical features, radiological, and histological findings in children is unknown.

Bronchiectasis not caused by cystic fibrosis (CF) is often perceived to be rare in western societies, but remains an important cause of chronic suppurative lung disease in the developing world..$^{3-5}$ A decline in hospital admission rates for paediatric bronchiectasis in the UK has been noted since the 1950s. ${ }^{67}$ This has been attributed to improved sanitation and nutrition, introduction of childhood immunisation, particularly against pertussis and measles, and the early and frequent use of antibiotics. ${ }^{8}$ Quantitative estimates of the prevalence of bronchiectasis in children worldwide are lacking. ${ }^{9}$

The advent of high resolution computed tomographic (HRCT) scanning, a highly sensitive non-invasive technique, has greatly improved our ability to recognise this condition and has now replaced bronchography as the "gold standard" imaging modality for diagnosing bronchiectasis. ${ }^{10}$ A correlation has been found between CT and pathological findings in adults with severe bronchiectasis requiring surgical resection of a lobe, with $87 \%$ sensitivity of CT diagnosis. ${ }^{10}$ There are no equivalent paediatric data.

In 1994 Nikolaizik and Warner estimated that 1\% of children referred for investigation of chronic respiratory symptoms had suppurative lung disease not resulting from CF. ${ }^{11}$ They established an underlying cause in $63 \%$ of cases, describing immunological abnormalities in $27 \%$, primary ciliary dyskinesia in $17 \%$, congenital malformation in $15 \%$, and aspiration in 5\% of cases. Diagnosis in this series was made using a variety of investigations including CT scanning. The epidemiology and aetiology has not been reviewed in a paediatric population since HRCT scanning was established as the gold standard radiological diagnosis in the late 1990s. It has been argued that chest radiography still has a role in the diagnosis of bronchiectasis in adults, but its diagnostic value in children is unknown. ${ }^{12}$

We report our local experience of HRCT defined non-CF bronchiectasis derived from a cohort of 93 children attending a tertiary paediatric respiratory centre at Freeman Hospital, Newcastle upon Tyne since 1996.

\section{METHODS}

A retrospective case note review was conducted of 93 consecutive patients with bronchiectasis confirmed by HRCT scanning. The children constituted $9.6 \%$ of all new referrals between November 1996 and May 2002. A radiological diagnosis of bronchiectasis was made on the HRCT scan if any of the following diagnostic criteria was present: ${ }^{10}$

- cross sectional diameter of one or more bronchi greater than that of the accompanying pulmonary artery;

- mucoid impaction within a dilated bronchus;

- non-tapering bronchi in cuts parallel to the direction of travel;

- bronchi visible adjacent to the non-mediastinal pleura.

The HRCT diagnosis was made by a consultant cardiothoracic radiologist who was aware of the clinical suspicion of bronchiectasis but not the site of any suspected abnormality. A chest radiograph report was obtained for each child, where 
available, taken up to a year before the HRCT scan. Most of the children were referred by paediatricians from district general hospitals or from primary care practices in the Northern Region. Children with primary immune deficiencies or following heart transplantation came from a wider geographical area.

Chest radiographs were reported by a number of different radiologists. As it was not possible to establish the individual criteria adopted by each radiologist for suspecting bronchiectasis, the reports were divided into three broad categories:

- normal;

- abnormal, with no report of bronchial dilation and not containing the phrase "suggestive of bronchiectasis";

- abnormal, with a report of bronchial dilation and/or containing the phrase "suggestive of bronchiectasis" with location specified.

All patients were investigated to determine the aetiology at the discretion of the attending paediatrician. Investigations included bronchoscopy, measurement of serum immunoglobulins (Igs), IgG subclasses, specific antibody responses to tetanus toxoid, and to the capsular polysaccharides of Haemophilus influenzae type b (Hib) and Streptococcus pneumoniae, and nasal brushings for ciliary beat frequency and electron microscopy. Bacterial culture of cough swab, sputum, or bronchoalveolar lavage (BAL) fluid was obtained in all patients. Sweat tests were performed in all cases unless bronchiectasis was limited to one lobe and there was an obvious associated clinical diagnosis, or if the child had received a cardiac transplant.

\section{RESULTS}

The male to female ratio of the 93 cases was $2: 1$. Table 1 shows their ages at symptom onset and establishment of the HRCT diagnosis of bronchiectasis. The median time to HRCT diagnosis from symptom onset was 3.0 years (range 0.1-14.8). This represents either a delay in presentation or in referral, as the median time to HRCT diagnosis from first tertiary centre appointment was only 0.2 years (range $0-4.5$ ).

The most common referral diagnosis of asthma in 45 cases (49\%, fig 1) was often reported to be "difficult to control". This diagnosis was felt to be incorrect on clinical review and lung function testing in 39 cases (87\%). Subsequent withdrawal of anti-asthma medication was not associated with clinical deterioration in any child. Table 2 shows the final associated diagnoses for the 93 children following investigation.

The most common association was of a previous pneumonic illness. This occurred before the age of 1 year in 16 of 34 cases $(47 \%)$. A familial syndrome occurred in two siblings in a family where two generations were affected by

Table 1 Median ages at HRCT diagnosis and symptom onset

\begin{tabular}{llll}
\hline & No $(\%)$ & Median & Range \\
\hline $\begin{array}{l}\text { Age of HRCT scan diagnosis } \\
\text { (years) }\end{array}$ & $92(100)$ & 7.2 & $1.6-18.8$ \\
$<5$ & $31(34)$ & & \\
$\geqslant 5-<10$ & $33(36)$ & & \\
$\geqslant 10$ & $28(30)$ & & \\
& & & \\
Age of symptom onset (years) & $81(100)$ & 1.1 & $0-16$ \\
$<5$ & $66(81)$ & & \\
$\geqslant 5-<10$ & $12(15)$ & & \\
$\geqslant 10$ & $3(4)$ & & \\
\hline
\end{tabular}

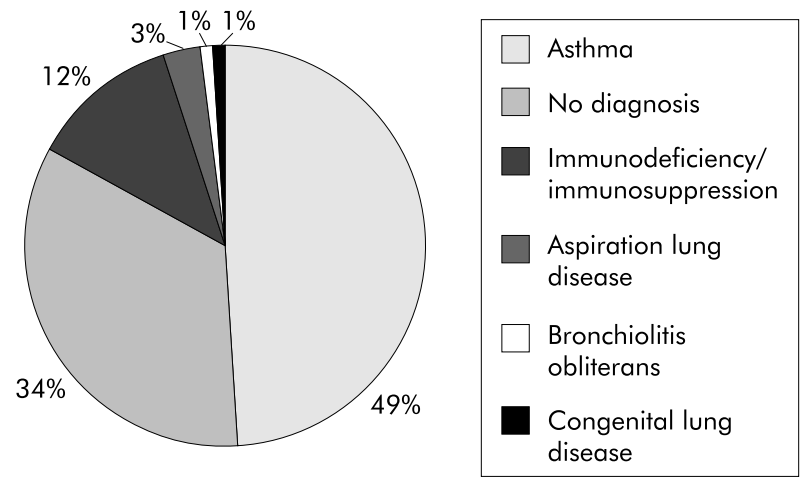

Figure 1 Referral diagnosis for 93 cases of non-CF bronchiectasis.

\begin{tabular}{|c|c|}
\hline Aetiology & No (\%) \\
\hline Post-pneumonic & 28130 \\
\hline Immunodeficiency/immunosuppression & $19(21)$ \\
\hline Idiopathic & $17(18$ \\
\hline Associated with bronchiolitis obliterans & $8(9)$ \\
\hline Congenital lung abnormality & $4(5)$ \\
\hline Chronic aspiration & $3(3)$ \\
\hline Post-pneumonic + congenital lung abnormality & 3 (3) \\
\hline Familial syndrome & $2(2)$ \\
\hline Post-pneumonic + immunodeficient & $2(2)$ \\
\hline $\begin{array}{l}\text { Hypersecretory asthma (right middle lobe } \\
\text { syndrome) }\end{array}$ & 1 (1) \\
\hline $\begin{array}{l}\text { Associated with primary eosinophilic } \\
\text { oesophagitis }\end{array}$ & $2(2)$ \\
\hline Primary ciliary dyskinesia & 1 (1) \\
\hline Immunodeficient + chronic aspiration & $2(2)$ \\
\hline Immunodeficient + congenital lung abnormality & 1 (1) \\
\hline
\end{tabular}

bronchiectasis in association with retinitis pigmentosa, in whom the genetic defect is unknown.

Major immune deficiency was the principle referring diagnosis for five children (chronic granulomatous disease in four and agammaglobulinaemia in one). Five children were immunosuppressed following cardiac transplantation and one child had been treated for acute lymphoblastic leukaemia. Immunoglobulins were measured in 80 children and five were shown to have an IgA deficiency. Of the 71 children for whom IgG subclasses were measured, 10 had an $\mathrm{IgG}_{2}$ deficiency and one had low $\operatorname{IgG}_{2}$ and $\mathrm{IgG}_{3}$ levels. Low levels of specific antibodies were detected in 55 children (75\%). A good response to appropriate vaccination occurred in 29 children. A primary diagnosis of specific antibody deficiency to capsulated bacteria was made in four cases and of common variable immune deficiency in one case.

Nasal ciliary beat frequency was measured in 46 children using the oscilloscopic technique ${ }^{13}$ and was within normal limits in 45. One child with Kartagener's syndrome was found to have primary ciliary dyskinesia.

Figure 2 shows the organisms isolated by standard bacterial culture techniques from cough swab, sputum, or BAL fluid. Each isolate was counted only once for each case to avoid the problem of multiple isolates of an organism during the same episode of illness. The organisms isolated most frequently were Haemophilus influenzae and Streptococcus pneumoniae. Cytomegalovirus was isolated in the BAL fluid of a neutropenic immunosuppressed patient. Pseudomonas aeruginosa was isolated infrequently. 


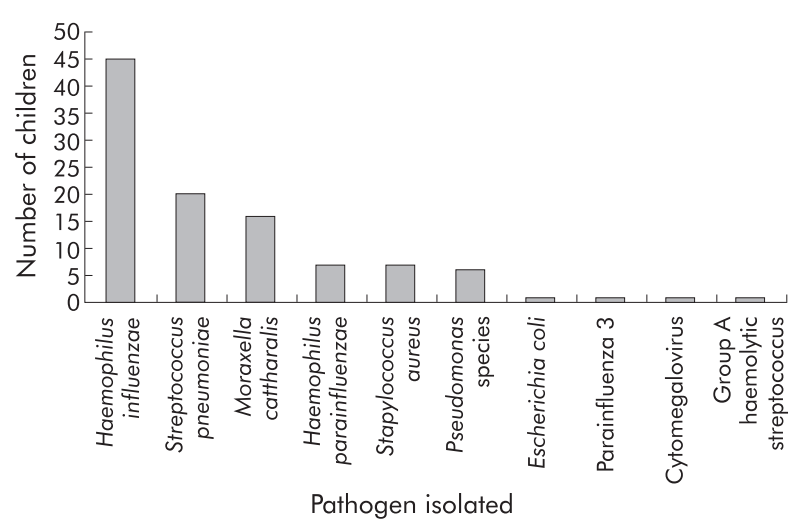

Figure 2 Respiratory pathogens isolated from 93 children with non-CF bronchiectasis.

Figure 3 illustrates the agreement between the chest radiography and HRCT reports in diagnosing bronchiectasis and the lobe(s) affected. In 12 children no radiography report was available in the year preceding the HRCT scan. The radiography report agreed exactly with the HRCT report in only five cases (5\%).

Repeat HRCT scans were performed in 18 cases a minimum of 18 months after HRCT diagnosis and initiation of treatment (range 18 months-5 years). The repeat scans were performed on clinical grounds. Complete radiological resolution of bronchiectasis on HRCT scan was reported in six cases (four post-pneumonic, two idiopathic) and in one case of post-pneumonic bronchiectasis the radiological appearance was improved. Radiological progression of the disease was reported in five cases (two immunocompromised, two postpneumonic, one hypersecretory asthma/right middle lobe syndrome) and the appearances were unchanged in the remaining six (two immunocompromised, one obliterative bronchiolitis, two post-pneumonic, one idiopathic).

\section{DISCUSSION}

HRCT defined non-CF bronchiectasis is not an uncommon problem in our referral population. The 93 cases described constitute $9.6 \%$ of all new referrals to a tertiary respiratory paediatric referral centre over a period of 5.5 years, which represents a 10 -fold higher rate of diagnosis than that described by Nikolaizik and Warner in $1994 .{ }^{11}$ This increase can largely be attributed to the introduction of HRCT scanning to investigate chronic respiratory illness in children

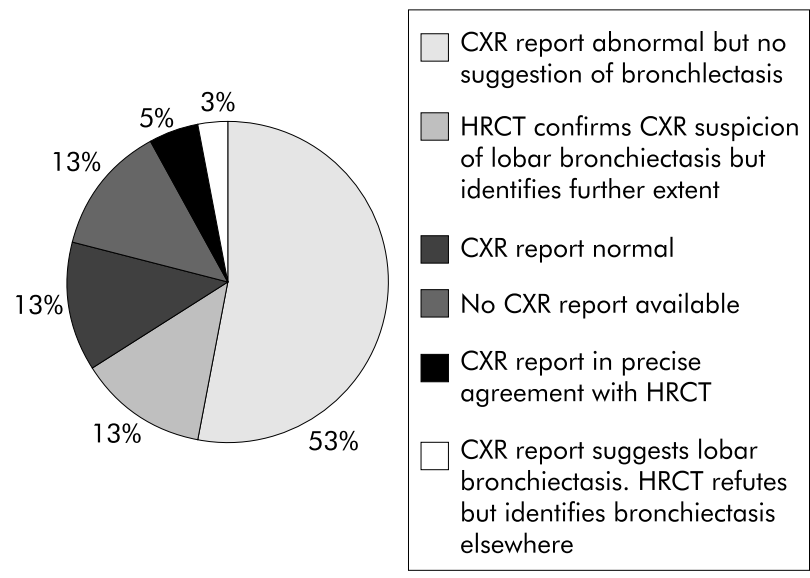

Figure 3 Agreement between chest radiograph (CXR) and HRCT scan for diagnosis of bronchiectasis and lobe(s) affected $(n=93)$. in recent years. A crude estimate of the prevalence of HRCT defined non-CF bronchiectasis in children under 17 years of age in the Northern Region (excluding South Cleveland) is one in 5800 based on the average annual birth rate of 31500 . It is acknowledged that this will include children at the milder end of the disease spectrum, as a single dilated bronchus of cross sectional diameter greater than that of its accompanying pulmonary artery is sufficient to satisfy the diagnostic criteria for HRCT bronchiectasis. It is anticipated, however, that there will also be a significant number of undiagnosed cases in the community.

Onset of respiratory symptoms is reported at a young age (median 1.1 years), but there is a pronounced delay from onset to diagnosis by HRCT scan (median 3.0 years). We believe that this may be due to the misdiagnosis of asthma in children with cough and no wheeze who had been labelled as having "cough variant asthma". Our findings support the assertion of McKenzie ${ }^{14}$ who has previously highlighted the potential inaccuracy of a diagnosis of asthma when based on the symptom of cough alone.

The chest radiography report was in exact agreement with the HRCT scan report for diagnosis and affected lobe(s) in only five cases (5\%). The chest radiograph was reported as normal in a further 12 children (13\%) with HRCT confirmed bronchiectasis. We acknowledge the limitations of comparison of radiographic reports made by multiple radiologists with an HRCT scan report made by a single cardiothoracic radiologist. We also accept that HRCT diagnosis of bronchiectasis is subject to some observer bias. Nevertheless, these findings suggest that the chest radiograph is of little diagnostic value in children with bronchiectasis, and that the report of a normal chest radiograph should not detract from further investigation of children with persistent respiratory symptoms who have evidence of chronic bacterial endobronchial infection.

Clarification of the diagnosis of bronchiectasis has major practical implications for the management of these patients. Revision of the diagnosis of asthma allows discontinuation of unnecessary medications with significant clinical and economic benefit. A diagnosis of bronchiectasis allows rational treatment with antibiotics and physiotherapy to be instituted. Studies in adults with bronchiectasis have shown that onset of sputum production commenced before the age of 10 years in $40 \%$ of cases, suggesting that the onset of disease is often in childhood..$^{15}$ Early diagnosis and treatment may improve the long term prognosis as it is known that aggressive treatment with antibiotics and physiotherapy slows disease progression of bronchiectasis in patients with CF and in children with primary ciliary dyskinesia. ${ }^{16}{ }^{17}$ We have also shown a resolution of the radiological changes of bronchiectasis on the HRCT scan in six cases following prompt management of respiratory exacerbations and regular physiotherapy a minimum of 18 months after initial diagnosis. HRCT resolution only occurred in patients in whom the aetiology was either non-progressive or idiopathic. These findings raise questions as to the nature of the relationship between radiological findings and the histological changes of bronchiectasis in children.

The pathogenesis of bronchiectasis is incompletely understood. The most commonly proposed pathophysiological mechanism is the "vicious cycle theory" whereby an initial insult damages the respiratory tract resulting in impaired mucociliary clearance. This leads to chronic bacterial infection associated with a persistent inflammatory response producing fibrotic changes. ${ }^{18}$ The initial trigger is often infective although other factors must also be considered, particularly those that predispose to bronchial and pulmonary infection including immunodeficiency and anatomical abnormalities of the airways. In this series of patients the 
Box 1 Proposed definitions for the components of chronic suppurative lung disease in childhood

\section{Pre-bronchiectasis}

Chronic or recurrent bacterial endobronchial infection which may be associated with non-specific changes such as may persist, resolve, or progress to "HRCT bronchiectasis".

\section{HRCT bronchiectasis}

The clinical features are associated with HRCT evidence of bronchial dilation. This entity may persist, progress to established bronchiectasis, return to a pre-bronchiectatic state, or resolve entirely.

\section{Established bronchiectasis}

The HRCT findings have not resolved after a significant time period (we suggest 2 years). This condition should then be regarded as irreversible. bronchial wall thickening on the HRCT scan. This condition

Normal

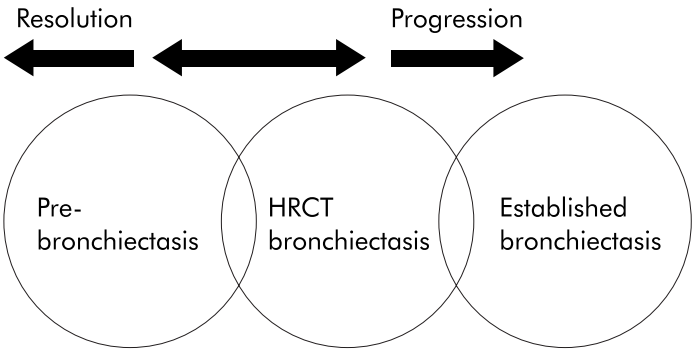

Figure 4 The spectrum of chronic suppurative lung disease of childhood.

\section{Authors' affiliations}

K M Eastham, Sir James Spence Institute of Child Health, Royal Victoria Infirmary, Newcastle upon Tyne NE1 4LP, UK

L Mitchell, Department of Radiology, Freeman Hospital, Newcastle upon Tyne NE7 7DN, UK

A J Fall, Department of Paediatrics, James Cook University Hospital, Middlesbrough, Cleveland TS4 3BW, UK

D A Spencer, Department of Respiratory Paediatrics, Freeman Hospital, Newcastle upon Tyne NE7 7DN, UK

\section{REFERENCES}

1 Lewiston NJ. Bronchiectasis in childhood. Pediatr Clin North Am 1984;31:865-78

most commonly reported initial insult appears to be a previous pneumonic illness which accounted for $30 \%$ of our cases. This is in contrast to $66-69 \%$ of cases in a series in the 1960s when conditions such as measles, pertussis and tuberculosis were more prevalent. ${ }^{19} 20$ It is known that a pneumonia occurring at the time of rapid lung development, during the first 3 years of life, may impair subsequent growth and lung function. ${ }^{21}{ }^{22}$ Sixteen $(47 \%)$ of our cases with a reported pneumonia experienced their illness before the age of 1 year, suggesting that immunological immaturity may be an important prognostic factor.

In our series $H$ influenzae and $S$ pneumoniae were isolated most commonly (fig 3 ). This differs from the bacteriological pattern seen in established bronchiectasis in adults where $P$ aeruginosa is a major pathogen. ${ }^{23}$

Low levels of specific antibodies were commonly encountered in this series; however, the good response to vaccines seen in the majority suggests that most children do not have a significant primary immunological problem. The relationship between low levels of bacterial antibodies and the bronchiectatic process remains uncertain.

It is clear from our series that the term "bronchiectasis", which describes a progressive and irreversible disease process, is often imprecise when referring solely to radiological changes in this age group. As the term bronchiectasis has both prognostic and therapeutic implications, we suggest that we should now attempt to improve the nomenclature of this group of disorders in children. We propose that chronic suppurative lung disease of childhood may be viewed as a disease spectrum comprising three interrelated components: pre-bronchiectasis, HRCT bronchiectasis, and established bronchiectasis (fig 4, box 1). Children with pre-bronchiectasis are presumably suffering from a form of chronic bronchitis, but we suggest that this term should be avoided as it differs from the classic form of smoking related chronic bronchitis seen in adults. Persistent endobronchial infection in the developing child appears to be associated with the subsequent development of bronchiectasis, whereas this does not appear to be the case for adult patients with smoking related chronic bronchitis.

Long term epidemiological studies will be required to establish details of the interrelationships and prognoses for these three disease entities.
2 Laënnec RTH. A treatise in the diseases of the chest and on mediate auscultation. 4th edition (1819). Translation by J Forbes. London: Longman, 1834.

3 Waite DA, Wakefield SJ, Moriarty KM, et al. Polynesian bronchiectasis. Eur J Respir Dis Suppl 1983;127:31-6.

4 Karakoc GB, Yilmaz M, Altintas DU, et al. Bronchiectasis: still a problem. Pediatr Pulmonol 2001;32:175-8.

5 Singleton R, Morris A, Redding G, et al. Bronchiectasis in Alaska native children: causes and clinical courses. Pediatr Pulmonol 2000;29:182-7.

6 Clark N. Bronchiectasis in childhood. BMJ 1963;1:80-7.

7 Field C. Bronchiectasis in childhood. III. Prophylaxis, treatment and progress with a follow-up study of 202 cases of established bronchiectasis. Pediatrics 1949;4:21-46.

8 Lewitson N. Bronchiectasis. In: Hilman B, ed. Pediatric respiratory disease. Philadelphia: WB Saunders, 1993:222-9.

9 Callahan CW, Redding GJ. Bronchiectasis in children: orphan disease or persistent problem? Pediatr Pulmonol 2002;33:492-6.

10 Kang EY, Miller RR, Muller NL. Bronchiectasis: comparison of preoperative thin-section CT and pathologic findings in resected specimens. Radiology 1995; 195:649-54.

11 Nikolaizik WH, Warner JO. Aetiology of chronic suppurative lung disease. Arch Dis Child 1994;70:141-2.

12 van der Bruggen-Bogaarts BA, van der Bruggen $H M$, van Waes PF, et al. Screening for bronchiectasis. A comparative study between chest radiography and high-resolution CT. Chest 1996;109:608-11.

13 Veale D, Rodgers AD, Griffiths CJ, et al. Variability in ciliary beat frequency in normal subjects and in patients with bronchiectasis. Thorax 1993;48:1018-20.

14 McKenzie S. Cough - but is it asthma? Arch Dis Child 1994;70:1-2.

15 Pasteur MC, Helliwell SM, Houghton SJ, et al. An investigation into causative factors in patients with bronchiectasis. Am J Respir Crit Care Med 2000;162:1277-84.

16 Ramsey BW, Pepe MS, Quan JM, et al. Intermittent administration of inhaled tobramycin in patients with cystic fibrosis. Cystic Fibrosis Inhaled Tobramycin Study Group. N Engl J Med 1999;340:23-30.

17 Ellerman A, Bisgaard $H$. Longitudinal study of lung function in a cohort of primary ciliary dyskinesia. Eur Respir J 1997;10:2376-9.

18 Cole P. The damaging role of bacteria in chronic lung infection. J Antimicrob Chemother 1997;40(Suppl A):5-10.

19 Glauser E, Cook C, Harris G. Bronchiectasis-a review of 187 cases in children with follow-up pulmonary function studies in 58. Acta Paediatr Scand 1966; 165(Suppl).

20 Strang C. The fate of children with bronchiectasis. Ann Intern Med 1956;44:630-56.

21 Castro-Rodriguez JA, Holberg CJ, Wright AL, et al. Association of radiologically ascertained pneumonia before age $3 \mathrm{yr}$ with asthmalike symptoms and pulmonary function during childhood: a prospective study. Am J Respir Crit Care Med 1999;159:1891-7.

22 Johnston ID, Strachan DP, Anderson HR. Effect of pneumonia and whooping cough in childhood on adult lung function. N Engl J Med 1998;338:581-7.

23 Nicotra MB, Rivera M, Dale AM, et al. Clinical, pathophysiologic, and microbiologic characterization of bronchiectasis in an aging cohort. Chest 1995; 108:955-61 